\title{
Right ventricular remodeling and dysfunction among the spectrum of heart failure phenotypes
}

\author{
Harris Wang ${ }^{1}$, Lingyu Xu ${ }^{1}$, Kelvin Chow ${ }^{2}$, Joseph J Pagano ${ }^{2}$, Anna Schmidtt, James A White ${ }^{3}$, \\ Evangelos Michelakis', Justin Ezekowitz ${ }^{1}$, Jason Dyck ${ }^{4}$, Mark Haykowsky ${ }^{4}$, Gavin Y Oudit ${ }^{1}$, Richard B Thompson ${ }^{2}$, \\ lan Paterson ${ }^{1 *}$
}

From 19th Annual SCMR Scientific Sessions

Los Angeles, CA, USA. 27-30 January 2016

\section{Background}

Right ventricular dysfunction (RVD) and enlargement (RVE) are increasingly associated with poor outcomes however their prevalence among patients with heart failure and those at risk has not been well characterized.

\section{Methods}

Healthy controls and patients with heart failure related structural heart disease underwent a standard cardiac magnetic resonance examination. Right and left ventricular ejection fraction (EF) and end diastolic volumes (EDV) were traced from cine imaging. RVD was defined as RVEF less than mean minus 2 standard deviations of healthy gender matched controls. RVE was defined as either indexed RVEDV or RVEDV/LVEDV exceeding mean plus 2 standard deviations of healthy gender matched controls. The prevalence of RV abnormalities and pattern of ventricular remodeling was compared among patient subgroups.

\section{Results}

89 healthy controls, age $57 \pm 10$ years, and 502 patients, age $56 \pm 16$ years, were included. Among controls, RVEF was $61 \pm 6 \%$ for women and $60 \pm 5.6 \%$ for men, $\mathrm{p}=0.5$, indexed RVEDV was $63 \pm 11 \mathrm{~mL} / \mathrm{m}^{2}$ for women and $71 \pm 12 \mathrm{~mL} / \mathrm{m}^{2}$ for men, $\mathrm{p}<0.05$, and mean RVEDV/LVEDV was $0.94 \pm 0.12$ for women and $0.98 \pm$ 0.14 for men, $\mathrm{p}=0.19$. Among patients, RVD was detected in $47 \%$, RVE in $38 \%$ and either RV abnormality in $61 \%$. RV abnormalities were common in all patient subgroups, ranging from $31 \%$ in hypertensive heart disease to $100 \%$ in right sided valvular heart disease.

${ }^{1}$ Cardiology, University of Alberta, Edmonton, AB, Canada

Full list of author information is available at the end of the article

\section{Conclusions}

RV dysfunction and/or enlargement are prevalent among patients with heart failure related structural heart disease. Routine assessment is therefore recommended in 3-D cardiac imaging.

\begin{tabular}{|c|c|c|c|c|}
\hline \multicolumn{5}{|c|}{ Table 1.Baseline Characteristics and CuR Weasures in $89 \mathrm{Heallthy}$ Controk } \\
\hline & $\begin{array}{c}\text { All } \\
(\mathrm{N}=89)\end{array}$ & $\begin{array}{l}\begin{array}{l}\text { Female } \\
(\mathrm{N}=42)\end{array} \\
\end{array}$ & $\begin{array}{c}\begin{array}{c}\text { Male } \\
(\mathrm{N}=47)\end{array}\end{array}$ & p-value ${ }^{*}$ \\
\hline Ageat Exam (yrs) & $57 \pm 10$ & $59 \pm 9$ & $55 \pm 11$ & 0.11 \\
\hline $\operatorname{BSA}\left(\mathrm{m}^{2}\right)$ & $1.9 \pm 0.2$ & $1.8 \pm 0.2$ & $2.0 \pm 0.2$ & $<0.001$ \\
\hline Systolic Blood Pressure (mmHg) & $130 \pm 15$ & $129 \pm 16$ & $131 \pm 14$ & 0.59 \\
\hline Diastolic Blood Pressure (mmHg) & 779 & $74 \pm 8$ & $81 \pm 9$ & $<0.001$ \\
\hline Heart Rate (bpm) & $68 \pm 10$ & $67 \pm 11$ & $68 \pm 9$ & 0.61 \\
\hline IVSV(mL) & $83 \pm 16$ & $74 \pm 13$ & $92 \pm 15$ & $<0.001$ \\
\hline Indexed LVSV $\left(\mathrm{ml} / \mathrm{m}^{2}\right)$ & $43 \pm 6$ & $41 \pm 5$ & $45 \pm 7$ & 0.002 \\
\hline IVEF(\%) & $62 \pm 4$ & $\overline{62 \pm 4}$ & $62 \pm 4$ & 0.988 \\
\hline LVEDV $(\mathrm{ml})$ & $135 \pm 28$ & $119 \pm 19$ & $148 \pm 27$ & $<0.001$ \\
\hline Indexed LVEDV $\left(\mathrm{mL} / \mathrm{m}^{2}\right)$ & $70 \pm 10$ & $66 \pm 7$ & $73 \pm 11$ & 0.001 \\
\hline LVESV(mL) & $51 \pm 13$ & $45 \pm 8$ & $57 \pm 14$ & $<0.001$ \\
\hline Indexed IVESV $\left(\mathrm{mL} / \mathrm{m}^{2}\right)$ & $27 \pm 5$ & $25 \pm 4$ & $28 \pm 6$ & 0.014 \\
\hline LV mass (g) & $102 \pm 26$ & $83 \pm 13$ & $119 \pm 22$ & $<0.001$ \\
\hline Indexed LV mass $\left(\mathrm{g} / \mathrm{m}^{2}\right)$ & $53 \pm 9$ & $46 \pm 6$ & $58 \pm 8$ & $<0.001$ \\
\hline RVSV(mL) & $78 \pm 17$ & $69 \pm 14$ & $85 \pm 15$ & $<0.001$ \\
\hline Indexed RVSV $\left(\mathrm{mL} / \mathrm{m}^{2}\right)$ & $40 \pm 7$ & $38 \pm 7$ & $42 \pm 7$ & $\overline{0.011}$ \\
\hline RVEF (\%) & $60 \pm 6$ & $61 \pm 6$ & $60 \pm 6$ & 0.50 \\
\hline RVEDV (mL) & $129 \pm 30$ & $113 \pm 24$ & $144 \pm 27$ & $<0.001$ \\
\hline Indexed RVEDV $\left(\mathrm{mL} / \mathrm{m}^{2}\right)$ & $67 \pm 12$ & $63 \pm 11$ & $71 \pm 12$ & 0.001 \\
\hline $\operatorname{RVESV}(\mathbf{m l})$ & $52 \pm 16$ & $45 \pm 12$ & $58 \pm 16$ & $<0.001$ \\
\hline Indexed RVESV $\left(\mathrm{mL} / \mathrm{m}^{2}\right)$ & $27 \pm 7$ & $25 \pm 6$ & $29 \pm 7$ & 0.007 \\
\hline LVmass/LVEDV (g/mL) & $0.76 \pm 0.11$ & $0.70 \pm 0.08$ & $0.81 \pm 0.10$ & $<0.001$ \\
\hline RVEDV/LVEDV & $0.96 \pm 0.13$ & $0.94 \pm 0.12$ & $0.98 \pm 0.14$ & 0.19 \\
\hline
\end{tabular}

Figure 1 All values are given as mean $+/$ - standard deviation except for number of subjects. * p-value of comparison between male and female healthy controls 


\begin{tabular}{|c|c|c|c|c|c|c|c|c|c|c|}
\hline \multicolumn{11}{|c|}{ Table 3. Demegraphlcs and Cardlac MR Measures by Dlaease Oroup } \\
\hline Dlsease Group & HTN & HFPEF & Infl|trative & Dllated & Ischemle & Myocarditis & $\begin{array}{l}\text { Valvular } \\
(L)\end{array}$ & $\begin{array}{l}\text { Valvular } \\
(R)\end{array}$ & $\mathrm{PH}$ & All \\
\hline Number (male) & $84(3 \theta)$ & $22(17)$ & $26(1 \theta)$ & $127(\theta 0)$ & $81(70)$ & $7 \theta(67)$ & $33(\theta)$ & $16(6)$ & $68(10)$ & 602(326) \\
\hline Ag* (yrs) & $83 \pm 12^{*}$ & $76 \pm 7^{*}$ & $64 \pm 14$ & $63 \pm 14^{*}$ & $8 \theta \pm 13^{*}$ & $42 \pm 14^{*}$ & $81 \pm 18$ & $63 \pm 10$ & $67 \pm 18$ & $68 \pm 16$ \\
\hline LVEF (\%) & $81 \pm 8$ & $68 \pm 7^{*}$ & $68 \pm 14$ & $32 \pm 14^{*}$ & $32 \pm 12^{*}$ & $48 \pm 14^{*}$ & $56 \pm 12^{*}$ & $80 \pm 11$ & $82 \pm 8$ & $47 \pm 17$ \\
\hline $\begin{array}{l}\text { Indexed LV mass } \\
\left(\mathrm{g} / \mathrm{m}^{2}\right)\end{array}$ & $86 \pm 13$ & $88 \pm 13$ & $84 \pm 32$ & $87 \pm 24$ & $83 \pm 23$ & $88 \pm 28$ & $73 \pm 25^{*}$ & $4 \theta \pm 13$ & $67 \pm 18$ & $74 \pm 23$ \\
\hline $\begin{array}{l}\text { Indexed LVEDV } \\
\left(m L \mathrm{~m}^{2}\right)\end{array}$ & $83 \pm 16$ & $88 \pm 17$ & $80 \pm 23$ & $12 \theta \pm 3 \theta$ & $112 \pm 44$ & $88 \pm 37$ & $104 \pm 44$ & $84 \pm 16$ & $82 \pm 18$ & $97 \pm 43$ \\
\hline $\begin{array}{l}\text { LVMAVEDV } \\
(g / \mathrm{mL})\end{array}$ & $\begin{array}{l}1.08 \pm \\
0.10\end{array}$ & $\begin{array}{l}1.04 \pm \\
0.20\end{array}$ & $\begin{array}{l}1.05 \pm \\
0.26\end{array}$ & $\begin{array}{l}0.88 \pm \\
0.16\end{array}$ & $\begin{array}{l}0.74 \pm \\
0.18\end{array}$ & $0.8 \pm 0.2$ & $\begin{array}{l}0.76 \pm \\
0.22\end{array}$ & $\begin{array}{c}0.79= \\
0.2\end{array}$ & $\begin{array}{l}0.05 \pm \\
0.23\end{array}$ & $\begin{array}{l}0.03= \\
0.24\end{array}$ \\
\hline RVEF (\%) & $68 \pm 8$ & $63 \pm 8$ & $62 \pm 14$ & $41 \pm 14$ & $48 \pm 14$ & $48 \pm 11$ & $63 \pm 12$ & $68 \pm 8$ & $41 \pm 12$ & $47 \pm 13$ \\
\hline $\begin{array}{l}\text { Indexed RVEDV } \\
\left(m \perp m^{2}\right)\end{array}$ & $83 \pm 14$ & $72 \pm 21$ & $77 \pm 17$ & $\theta 2 \pm 30$ & $80 \pm 28$ & $86 \pm 28$ & $80 \pm 28$ & $108 \pm 33$ & ${ }_{32}^{103 \pm}$ & $88 \pm 29$ \\
\hline RVEDVILVEDV & $\begin{array}{l}1.01 \pm \\
0.16\end{array}$ & $\begin{array}{l}1.08= \\
0.24\end{array}$ & $\begin{array}{l}1.01 \pm \\
0.28\end{array}$ & $\begin{array}{l}0.74 \pm \\
0.23\end{array}$ & $\begin{array}{l}0.7 \theta \pm \\
0.38\end{array}$ & $0.9 \theta \pm 0.24$ & $\begin{array}{l}0.86 \pm \\
0.32\end{array}$ & $\begin{array}{l}1.7 \pm \\
0.46\end{array}$ & $\begin{array}{l}1.78 \pm \\
0.88\end{array}$ & $0.99 \pm$ \\
\hline RVD & $12(10 \%)$ & $8(38 \%)$ & $\theta(38 \%)$ & $\begin{array}{c}84 \\
(88 \%)\end{array}$ & $40(48 \%)$ & $33(42 \%)$ & $10(30 \%)$ & $3(20 \%)$ & $\begin{array}{c}38 \\
(88 \%)\end{array}$ & $\begin{array}{c}237 \\
(47 \%)\end{array}$ \\
\hline RVE & $\theta(14 \%)$ & $6(23 \%)$ & $7(28 \%)$ & $\begin{array}{c}62 \\
(41 \%)\end{array}$ & $18(22 \%)$ & $28(36 \%)$ & $8(24 \%)$ & $\begin{array}{c}16 \\
(100 \%)\end{array}$ & $47(84 \%)$ & $\begin{array}{c}109 \\
(38 \%)\end{array}$ \\
\hline RVD or RVE & $20(31 \%)$ & $11(50 \%)$ & $11(44 \%)$ & $\begin{array}{c}94 \\
(74 \%)\end{array}$ & $41(51 \%)$ & $4 \theta(82 \%)$ & $\begin{array}{c}14 \\
(42 \%)\end{array}$ & $\begin{array}{c}16 \\
(100 \%)\end{array}$ & $\begin{array}{c}50 \\
(89 \%)\end{array}$ & $\begin{array}{l}308 \\
(81 \%)\end{array}$ \\
\hline
\end{tabular}

Figure 2 All values are given as mean $+/$ - standard deviation except for number of subjects.

\section{Authors' details}

${ }^{1}$ Cardiology, University of Alberta, Edmonton, AB, Canada. ${ }^{2}$ Biomedical Engineering, University of Alberta, Edmonton, $A B$, Canada. ${ }^{3}$ University of Calgary, Calgary, AB, Canada. ${ }^{4}$ University of Alberta, Edmonton, AB, Canada.

Published: 27 January 2016

doi:10.1186/1532-429X-18-S1-P73

Cite this article as: Wang et al: Right ventricular remodeling and

dysfunction among the spectrum of heart failure phenotypes. Journal of Cardiovascular Magnetic Resonance 2016 18(Suppl 1):P73.
Submit your next manuscript to BioMed Central and take full advantage of:

- Convenient online submission

- Thorough peer review

- No space constraints or color figure charges

- Immediate publication on acceptance

- Inclusion in PubMed, CAS, Scopus and Google Scholar

- Research which is freely available for redistribution 